SMI-5-93

May, 1993

\title{
Large N QCD at High Energies as Two-Dimensional Field Theory
}

\author{
I.Ya. Aref'eva * \\ Steklov Mathematical Institute, \\ Russian Academy of Sciences, \\ Vavilov st.42, GSP-1,117966, \\ Moscow, Russia
}

\begin{abstract}
Different aspects of the Verlinde and Verlinde relation between high-energy effective scattering in QCD and a two-dimensional sigma-model are discussed. Starting from a lattice version of the truncated 4-dimensional Yang-Mills action we derive an effective theory with non-trivial longitudinal dynamics which has a form of the lattice two-dimensional chiral field model with non-trivial boundary conditions. To get quantum corrections coming from non-trivial longitudinal dynamics to transversal high-energy effective action one has to solve the twodimensional chiral field model with non-trivial boundary conditions. We do this within an approximation scheme which takes into account one-dimensional excitations. Contributions of the one-dimensional excitations to quantum corrections for the high-energy effective action are calculated in the large $\mathrm{N}$ limit using the character expansion method.
\end{abstract}

*E-MAIL: Arefeva@qft.mian.su 


\section{Introduction}

One of the striking results of intensive studies of high-energy scattering in QCD [1, 2] is that the scattering amplitudes are related with two-dimensional field theory [3]. Recently H.Verlinde and E. Verlinde have formulated a simple model in which this twodimensional nature of the interactions is manifest [4]. They have also shown that their formulation is in agreement with known results from standard perturbation theory [1, 2].

There are some questions concerning the Verlinde and Verlinde approach which we would like to address in this paper. First question is related with ultra-violet divergences and renormalizations. The Verlinde and Verlinde effective action which describes the transversal dynamics has a form of the two-dimensional $\sigma$-model. In spite of a non-convention form of this $\sigma$-model it is reasonable to expect that it is asymptotic free. But the $\beta$-functions corresponding to the 4-dimensional Yang-Mills theory and the two-dimensional $\sigma$-model numerically have the different forms. Namely, the 4dimensional Yang-Mills $\beta$-function contains the factor $\pi$ and $\beta$-function of the twodimensional $\sigma$-model does not. So one can expect that an additional renormalization comes from the longitudinal dynamics. This circumstance give us a raison to study the longitudinal dynamics more carefully. A treatment of longitudinal dynamics within the framework of the initial truncated Yang-Mills action is a main goal of this paper.

To analyze quantum corrections to the longitudinal dynamics and the corresponding renormalization we have to introduce some regularization. As a regularization we can introduce a lattice in x-space. Recall that one can deal with 4-dimensional lattice only in the Euclidean space-time. However, the high-energy effective action has been obtained [4] in the Minkowski space-time. The Minkowski signature is essential, since it permits to use the property of the two-dimensional wave equation. We would like to know does a similar action arise in the Euclidean formulation and what are its

quantum corrections. For this purpose we study the lattice version of the truncated action. It turns out that the lattice version of the truncated action looks like the usual lattice version of the two-dimensional chiral model with non-trivial boundary conditions. An interaction between fields living on the transversal planes appears due to the boundary effects in the two-dimensional chiral model. The lattice two-dimensional chiral model with non-trivial boundary conditions cannot be solved exactly. One can consider the one-dimensional chiral model and analyze the form of the corresponding transversal effective action. As in the two-dimensional case we can speculate that the one-dimensional answer describes the contribution coming from long one-dimensional excitations. In other words one can estimate long tubes contribution to the quantum version ofVV effective action.

The paper is organized as follows. In section 2 we present a brief review of the Verlinde and Verlinde results and write down a lattice version of the truncated action. In section 3 we study the longitudinal dynamics for a model case then this dynamics is one-dimensional and calculate a leading term of the high-energy effective action for large N. We discuss the two-dimensional longitudinal dynamics in a concluding section. 


\section{Lattice Effective Action for QCD at High Ener- gies.}

\subsection{Truncated Action}

Since this paper is inspired by the recent H.Verlinde and E.Verlinde paper [4], let us start from a brief presentation of their results. They are interested in the behaviour of scattering amplitudes in the kinematical regime where $s$ is much larger than $t$, while $t$ is also larger than the QCD scale $\Lambda_{q c d}$. Introducing two light-cone coordinates $x^{\alpha}=\left(x^{+}, x^{-}\right)$with $x^{ \pm}=x \pm t$ and two transverse coordinates $z^{i}=(y, z)$, authors assume that two fast moving particles have very large momenta in the $x^{ \pm}$direction, while they remain at a relatively large distance in the $z$-direction. They also use the hypothesis, according to which the typical longitudinal momentum of the dynamical modes in this process grows proportionaly to the centre of mass energy, whereas the typical size of the transversal momenta is determined by the momentum transfer. A similar assumption was used also in other approaches to high energy QCD [1]. The distinguished feature of the H.Verlinde and E.Verlinde approach is that the high-energy limit is taken directly at the level of the action.

Let us do this for the pure Yang-Mills model, described by

$$
S=\frac{1}{4 g^{2}} \int d^{4} x \operatorname{tr}\left(F_{\mu \nu} F^{\mu \nu}\right) .
$$

Here $F_{\mu \nu}$ is the non-abelian field strength, $F_{\mu \nu}=\partial_{\mu} A_{\nu}-\partial_{\nu} A_{\mu}+\left[A_{\mu}, A_{\nu}\right], A_{\mu}=A_{\mu}^{a} \tau^{a}$ where $g$ is the coupling constant and $\tau^{a}$ are the generators of the Lie algebra of the gauge group $G=S U(N)$.

The main idea [4] is that by performing a rescaling of the longitudinal coordinates $x^{\alpha} \rightarrow \lambda x^{\alpha}$ inside the Yang-Mills action (11), one can see which part will become strongly or weakly coupled when one looks at the theory at high longitudinal energies. The components of the gauge potential transform under this rescaling as $A_{i} \rightarrow A_{i}$, while $A_{\alpha} \rightarrow \lambda^{-1} A_{\alpha}$ and the rescaled Yang-Mills action can be written as

$$
S_{Y M}^{\prime}=\frac{1}{4 \lambda^{2} g^{2}} \int \operatorname{tr}\left(F^{\alpha \beta} F_{\alpha \beta}\right)+\frac{1}{2 g^{2}} \int \operatorname{tr}\left(F^{\alpha i} F_{\alpha i}\right)+\frac{\lambda^{2}}{4 g^{2}} \int \operatorname{tr}\left(F_{i j} F^{i j}\right) .
$$

Introducing an auxiliary field $E_{\alpha \beta}=-E_{\beta \alpha}$ one can rewrite (2) in the form

$$
S_{Y M}^{\prime}=\frac{1}{2 g^{2}} \int \operatorname{tr}\left(E^{\alpha \beta} F_{\alpha \beta}+F_{\alpha i} F^{\alpha i}\right)+\frac{\lambda^{2}}{4 g^{2}} \lambda^{2} \int \operatorname{tr}\left(E_{\alpha \beta} E^{\alpha \beta}+F_{i j} F^{i j}\right) .
$$

The description of a scattering process with some $s$ and $t$ using the standard action is completely equivalent to that using the rescaled action (3) with rescaled $s$ to $s^{\prime}=\lambda^{2} s$, i.e.

$$
\mathcal{A}\left(S_{Y M}, s\right)=\mathcal{A}\left(S_{Y M}^{\prime}, \lambda^{2} s\right),
$$

where $\mathcal{A}$ is a scattering amplitude describing the process. One can use this correspondence and reformulate the high-energy limit $s \rightarrow \infty$ in QCD as the $\lambda \rightarrow 0$ limit of the rescaled theory (3) with $s^{\prime}$ fixed, i.e. $\lambda \sim \frac{1}{\sqrt{s}} \rightarrow 0$. 
Therefore, high-energy scattering in gauge theories can be studied by means of the truncated Yang-Mills action

$$
S[A, E]=\frac{1}{2 g^{2}} \int \operatorname{tr}\left(E^{\alpha \beta} F_{\alpha \beta}+F_{\alpha i} F^{\alpha i}\right) .
$$

The auxiliary field $E_{\alpha \beta}$ can be integrated out yielding an effective theory describing the zero-curvature longitudinal gauge-field $A_{\alpha}$ of the form

$$
A_{\alpha}=\partial_{\alpha} V V^{+}
$$

Here the group element $V$ may still depend on all four coordinates I. Substituting (6) into the action $S$, one gets

$$
S\left[V, A_{i}\right]=\frac{1}{2 g^{2}} \int \operatorname{tr}\left(\partial_{\alpha}\left(V^{+} D_{i} V\right)\right)^{2}, \quad D_{i}=\partial_{i}+A_{i}
$$

At the formal level the longitudinal components $A_{\alpha}$ are completely eliminated by a gauge transformation of $A_{i}$ to $\widetilde{A}_{i}=V^{-1} D_{i} V$, i.e.

$$
S\left[V, A_{i}\right]=\frac{1}{2 g^{2}} \int \operatorname{tr}\left(\partial_{\alpha} \widetilde{A}_{i}\right)^{2}
$$

However if we consider an interaction of gauge fields with charged particles, then nonarbitrary gauge transformations at infinity are allowed.

\subsection{Lattice Truncated Action}

Another subtle question related to the limit $\lambda \rightarrow 0$ is the problem of divergences. To avoid ultra-violet divergences one can put the theory on the lattice. Let us consider a lattice version of the rescaled action (2)

$$
\begin{gathered}
S_{L Y M}^{\prime}=\frac{1}{4 \lambda^{2} g^{2}} \sum_{x} \sum_{\alpha, \beta}\left(\operatorname{tr} U\left(\square_{\alpha, \beta}\right)-1\right)+\frac{1}{4 g^{2}} \sum_{x} \sum_{\alpha, i}\left(\operatorname{tr} U\left(\square_{\alpha, i}\right)-1\right) \\
+\frac{\lambda^{2}}{4 g^{2}} \sum_{x} \sum_{i, j}\left(\operatorname{tr} U\left(\square_{i, j}\right)-1\right) .
\end{gathered}
$$

Here $x$ are the points of the 4-dimensional lattice, $\alpha, \beta$ are unit vectors in the longitudinal direction and $i, j$ are unit vectors in the transversal direction; $\square_{\mu, \nu}$ is a single plaquette attached to the links $(x, x+\mu)$ and $(x, x+\nu), \mu, \nu=\alpha, i$. Performing the $\lambda \rightarrow 0$ limit in the rescaled lattice theory (9) we get a lattice version of the truncated Lagrangian (7)

$$
S_{L Y M}^{\prime}=\frac{1}{4 g^{2}} \sum_{x} \sum_{\alpha, i} \operatorname{tr}\left(U\left(\square_{\alpha, i}\right)-1\right),
$$

with $U_{x, \alpha}$ being a subject of the relation

$$
U\left(\square_{\alpha, \beta}\right)-1=0,
$$

\footnotetext{
${ }^{1}$ Jacobian of this replacement of the variables $E^{\alpha \beta}$ and $A_{\alpha}$ by $U$ is simply equal to 1 《]
} 
i.e. $U_{x, \alpha}$ is a zero-curvature lattice gauge field

$$
U_{x, \alpha}=V_{x} V_{x+\alpha}^{+}
$$

Substituting (12) in (10) we get

$$
S_{t r}=\frac{1}{4 g^{2}} \sum_{x} \sum_{\alpha, i} \operatorname{tr}\left(V_{x+\alpha}^{+} U_{x+\alpha, i} V_{x+\alpha+i} V_{x+i}^{+} U_{x, i}^{+} V_{x}-1\right) .
$$

Eliminating $V_{x}$ by the gauge transformation $U_{x, i} \rightarrow \tilde{U}_{x, i}=V_{x}^{+} U_{x, i}^{+} V_{x+i}$ we get the lattice version of the Lagrangian (9), i.e.

$$
S_{t r}=\frac{a^{D-4}}{4 g^{2}} \sum_{x} \sum_{\alpha, i} \operatorname{tr}\left(U_{x, i} U_{x+\alpha, i}^{+}-1\right) .
$$

Let us denote the points of 4-dimensional lattice as $x=(y, z)$, where $y$ and $z$ are the points of two two-dimensional lattices, say, y-lattice (longitudinal) and z-lattice (transversal). We see that the action (14) has a factorisation form

$$
S_{t r}=\frac{a^{D-4}}{4 g^{2}} \sum_{i, z} \sum_{y, \alpha} \operatorname{tr}\left(U_{y, z ; i} U_{y+\alpha, z ; i}^{+}-1\right) .
$$

Here we restore for the moment a factor $a^{D-4}$, which is omitted in the previous formula since we assumed $D=4$. The indices $z$ and $i$ play a role of isotopic indices. Let $(L)^{2}$ is a number of points of the z-lattice. Then we can say that we have $2(L)^{2}$-copies of two-dimensional lattice models. Each model describes the chiral field $U_{y}$, attached to the sites of the two-dimensional $y$-lattice

$$
\tilde{S}_{t r}=\frac{1}{4 g^{2}} \sum_{y, \alpha} \operatorname{tr}\left(U_{y} U_{y+\alpha}^{+}-1\right) .
$$

If one forgets about subtlety with the boundary conditions then for trivial boundary conditions one can say that the free energy per unit volume of the model (14) can be represented as

$$
E(N, g(a), a)=\frac{1}{V o l_{4}} \ln \left[\int \exp \left(S_{t r}\right) \prod_{y, z, i} d U_{y, z, i}\right]=\frac{2}{V o l_{2}} \ln \left[\int \exp \left(\tilde{S}_{t r}\right) \prod_{y} d U_{y}\right]
$$

Note that there is some similarity between $\lambda$ rescaling and the high temperature limit of lattice gauge theory [7], in the first case one makes a decomposition $(2, d-2)$ and in the second $(1, d-1)$.

The classical continuous limit of the action (16) gives the usual two-dimensional chiral model

$$
S_{t r, c l}=\frac{1}{4 g^{2}} \int d^{2} x \operatorname{tr}\left(\partial_{\alpha} U(x) \partial_{\alpha} U^{+}(x)\right) .
$$

Therefore, removing two-dimensional lattice by $a \rightarrow 0$ limit in (14) one gets $(L)^{2(D-2)_{-}}$ copies of the two-dimensional continuous models. 
To remove the $y$-lattice in the quantum version of the two-dimensional chiral model one has to perform the renormalization. In the one-loop approximation [5] one has

$$
\frac{1}{g^{2}(a)}=\frac{1}{g^{2}(\mu)}+c_{c h} \log (a \mu)^{1}
$$

with

$$
c_{c h}=N
$$

Note that to remove a lattice in the regularized version of the quantized Yang-Mills theories one has to perform the renormalization

$$
\frac{1}{g^{2}(a)}=\frac{1}{g^{2}(\mu)}+c_{Y M} \log (a \mu)^{1}
$$

with

$$
c_{Y M}=\frac{1}{16 \pi^{2}} \frac{11 N}{3} .
$$

Both theories are asymptotically free, but the renormalizations of coupling constants do not coincide. This means that taking the limit $\lambda \rightarrow 0$ we drop diagrams which contribute to the renormalization of the coupling constant.

Note also that (14) does not give a usual 4-dimensional model in the limit $a \rightarrow 0$, since we have not an extra factor $(a)^{2}$ which would lead us to 4 -dimensional continuous theory.

Let us compare the Lagrangian (14) with the Lagrangian (8). Assuming that $U_{x, i}$ has a form $U_{x, i}=\exp a A_{i}\left(x_{ \pm}, z\right)$ we reproduce (8), since extra factor $a^{2}$ together with summation over the z-lattice produces the integral over the z-plane. At first sight there are no raisons to get propagator modes in the z-plane, since there are not the corresponding kinetic terms. The Verlinde and Verlinde have observed that a non-trivial dynamics in the transversal z-plane can be introduced due to non-trivial boundary conditions in the $x_{ \pm}$-plane. Namely, they rewrote the action (7) as

$$
S\left[U, A_{i}^{c l}\right]=\frac{1}{2 e^{2}} \int d^{2} z \operatorname{tr}\left[\int_{-\infty}^{\infty} d x^{+} \partial_{+}\left(U^{-1} D_{i} U\right) \times \int_{-\infty}^{\infty} d x^{-} \partial_{-}\left(U^{-1} D_{i} U\right)\right]
$$

and then expressed the right-hand side directly in terms of the asymptotic values for $U$ and $A_{i}$ as follows

$$
\begin{gathered}
\int_{-\infty}^{\infty} d x^{-} \partial_{-}\left(U^{-1} D_{i} U\right)=g_{2}^{+} D_{i}^{+} g_{2}-g_{1}^{+} D_{i}^{+} g_{1} \\
\int_{-\infty}^{\infty} d x^{+} \partial_{+}\left(U^{-1} D_{i} U\right)=h_{2}^{-1} D_{i}^{-} h_{2}-h^{-1} D_{i}^{-} h_{1}
\end{gathered}
$$

where the covariant derivatives $D_{i}^{ \pm}$on the right-hand sides are with respect to $a_{i}^{ \pm}$. The effective action of the two-dimensional variables $\left(g_{A}, h_{B}, A_{i}^{ \pm}\right)$they represented as

$$
S\left[g_{A}, h_{B}, A_{i}^{ \pm}\right]=\frac{1}{2 g^{2}} \int d^{2} z M^{A B} \operatorname{tr}\left(g_{A}^{+} D_{i}^{+} g_{A} h_{B}^{+} D_{i}^{-} h_{B}\right),
$$


where $M^{A B}$ is the $2 \times 2$ matrix, $M^{A B}=\left(\begin{array}{cc}1 & -1 \\ -1 & 1\end{array}\right)$ and the indices $A(B)=1,2$ are summed over. In [4] was argued that the matrix $M^{A B}$ should be regularized as

$$
M_{r e g}^{A B}=\left(\begin{array}{cc}
1+\epsilon & -1+\epsilon \\
-1+\epsilon & 1+\epsilon
\end{array}\right)
$$

where

$$
\epsilon^{-1}=1-\frac{2 i}{\pi} \log s .
$$

Note that to get the representation (24) one has to deal with the Minkowski signature. Working with the usual lattice approximation, i.e. dealing with the Euclidean signature one cannot get in the classical approximation an analog of the representation (24) typical for solutions of the wave equation.

\section{One-dimensional Longitudinal Dynamics}

Now let us discuss effects related with non-trivial boundary conditions in the action (15). It is rather instructive to consider a model example of the one-dimensional ylattice. So, we deal with the following lattice integrals

$$
Z_{1+2}=\int \exp \left\{\frac{a N}{4 g^{2}} \sum_{i, z} \sum_{y} \operatorname{tr}\left(\tilde{U}_{y, z ; i} \tilde{U}_{y+1, z ; i}^{+}+\tilde{U}_{y+1, z ; i} \tilde{U}_{y, z ; i}^{+}-2\right)\right\} \prod_{i, z, y} d U_{y, z ; i} d V_{z ; i}
$$

More precisely we mean the following boundary conditions

$$
\begin{gathered}
Z_{1+2}=\int \prod_{(z, i)}\left(\int \exp \left\{\frac{a N}{4 g^{2}} \sum_{y} \operatorname{tr}\left(\tilde{U}_{y, z ; i} \tilde{U}_{y+1, z ; i}^{+}+\tilde{U}_{y+1, z ; i} \tilde{U}_{y, z ; i}^{+}-2\right)\right\} .\right. \\
\times \quad \prod_{\tilde{U}_{0, z ; i}}=V_{0, z} U_{0, z ; i} V_{0, z+i}^{+} \\
\tilde{U}_{L, z ; i}=V_{L, z} U_{L, z ; i} V_{L, z+i}^{+}
\end{gathered}
$$

In (27) we have the product of the transition functions of the one-dimensional lattice chiral model. This correlation function can be calculated [6] using the characters expansion method,

$$
\begin{gathered}
\mathcal{K}\left(U_{0}, U_{L}\right)=\int \exp \left\{N \tilde{\beta} \sum_{n=0}^{L} \operatorname{tr}\left(U_{n} U_{n+1}^{+}+U_{n+1} U_{n}^{+}-2\right)\right\} \prod_{n=1}^{L-1} d U_{n} \\
=\sum_{R} \operatorname{dim}_{R}\left(Z_{R}\right)^{(L-1)} \chi_{R}\left(U_{0} U_{L}^{+}\right)
\end{gathered}
$$

where $\chi_{R}$ and $\operatorname{dim}_{R}=\chi_{R}(I)$ are characters and dimensions of the $\mathrm{R}$ 's irreducible representation respectively. Here $L^{\prime}=L-1$. $Z_{R}$ are given by the following formula

$$
Z_{R}=\frac{1}{\operatorname{dim}_{R}} \int d U \chi_{R}\left(U^{+}\right) \exp \left\{N \tilde{\beta} \operatorname{tr}\left(U+U^{+}-2\right)\right\}
$$


where $\tilde{\beta}=a^{D-4} / 4 g^{2}$. The representation $\mathrm{R}$ of $\mathrm{U}(\mathrm{N})$ can be labelled by $\mathrm{N}$ integers $r=\left(n_{1}, n_{2}, \ldots, n_{N}\right)$ with $n_{1} \geq n_{2} \geq \ldots \geq n_{n}$, where $n_{i}$ corresponds to the number of boxes in the i-th row of a Young tableau. The explicit expression for the character $\chi_{R}(U)$ in terms of the eigenvalues $e^{i \phi_{k}}, 0 \leq \phi_{k} \leq 2 \pi$, of matrix $U$ is given by the Weyl formula [8]

$$
\chi_{\left(n_{1}, n_{2}, \ldots, n_{N}\right)}(U)=\frac{\operatorname{det}\left[e^{i\left(N-j+n_{j}\right) \phi_{k}}\right]}{\operatorname{det}\left[e^{i(N-j) \phi_{k}}\right]}
$$

where the quantities insides the det are to be viewed as the $j k$ elements of a matrix $M_{i k}$. However, for our purpose we need the explicit dependence on the matrix $U$ rather than on its eigenvalues. Such formulas are given by the well-known Frobenius relation 8] between characters and symmetric polynomials,

$$
\chi_{R}(U)=\sum_{\sigma \in S_{n}} \frac{\chi_{R}(\sigma)}{n !} \prod_{j=1}^{K_{\sigma}} \operatorname{tr}(U)^{k_{j}}
$$

where $n$ is a number of boxes in the Young tableau corresponding to the representation $\mathrm{R}$ of $\mathrm{SU}(\mathrm{N}), S_{n}$ is the symmetric group on n objects. For a given permutation $\sigma K_{\sigma}$ is a total number of cycles and $\left\{k_{1}, \ldots k_{j}, \ldots k_{K_{\sigma}}\right\}$ are cycle lengths. In the right-hand side of (31) $\operatorname{tr} U$ is trace in the fundamental representation. For the first characters one find explicit formula in [9, 10].

The integrals $Z_{R}$ (29) for the characters corresponding to the single row Young tableau with $\mathrm{n}$ boxes can be written in the form

$$
Z_{(n, 0,0, \ldots)}=\int d U \int_{0}^{2 \pi} \frac{d \phi}{2 \pi} \frac{e^{-i n \phi}}{\operatorname{det}\left(1-e^{i \phi} U\right)} e^{N \tilde{\beta} \operatorname{tr}\left(U+U^{+}-2\right)} .
$$

$Z_{0} \equiv Z_{(0, \ldots)}$ has the representation as a determinant of Bessel function [9]

$$
Z_{0}(N \tilde{\beta}, N)=e^{-2 N^{2} \tilde{\beta}} \operatorname{det}\left|I_{i-j}(2 N \tilde{\beta})\right| .
$$

$Z_{0}(N \tilde{\beta}, N)$ is nothing but the partition function of the Gross and Witten model [11] and it exhibits the phase transition

$$
Z_{0}(N \tilde{\beta}, N) \underset{N \rightarrow \infty}{\sim} \begin{cases}\exp \left\{-N^{2}\left(\frac{3}{4}+\frac{1}{2} \ln 2 \tilde{\beta}\right)\right\} & \text { if } \tilde{\beta}>1 / 2 \\ \exp \left\{-N^{2} \tilde{\beta}(2-\tilde{\beta})\right\} & \text { if } \tilde{\beta} \leq 1 / 2\end{cases}
$$

$Z_{(1,0,0 \ldots)}(N \tilde{\beta}, N)=\frac{Z_{0}}{N}<\operatorname{tr} U>$, can be found from the relation

$$
<\operatorname{tr} U>=N+\frac{1}{2 N} \frac{\partial}{\partial \tilde{\beta}} \log Z_{0}(N \tilde{\beta}, N)
$$

where

$$
<f>=\frac{\int d U f(U) \exp \left\{N \tilde{\beta} \operatorname{tr}\left(U+U^{+}-2\right)\right\}}{\int d U \exp \left\{N \tilde{\beta} \operatorname{tr}\left(U+U^{+}-2\right)\right\}}
$$

We have

$$
\frac{1}{N}<\operatorname{tr} U>=f(\tilde{\beta})
$$


where

$$
f(\tilde{\beta}) \underset{N \rightarrow \infty}{\sim} \begin{cases}1-\frac{1}{4 \tilde{\beta}} & \text { if } \tilde{\beta}>1 / 2 \\ \tilde{\beta} & \text { if } \tilde{\beta} \leq 1 / 2\end{cases}
$$

The explicit representations for the first $Z_{R}$ 's can be found from the explicit representations (??)-(??) and the generating functional

$$
Z\left(A, A^{+}\right)=\int d U e^{N \tilde{\beta} \operatorname{tr}\left(U A^{+}+U^{+} A\right)}
$$

The integral (39) defines the well known object, this is the partition function of the Brezin-Gross model which describes one link gauge field in the external matrix source 12.

Let us examine the behaviour of $Z_{R}$ in the large $N$ limit. Substituting the Frobenious relation (31) for the characters (31) in (29),

$$
\mathcal{Z}_{R_{Y_{n}}}=\sum_{\sigma \in S_{n}} \frac{\chi_{R}(\sigma)}{n !} \int \prod_{j=1}^{K_{\sigma}} \operatorname{tr}(U)^{k_{j}} \exp \left\{N \tilde{\beta} \operatorname{tr}\left(U+U^{+}-2\right)\right\} d U
$$

we see that different permutations $\sigma$ give different powers of $\mathrm{N}$ in the integral over $\mathrm{U}$. The main contribution comes from $\sigma$ with the most number of cycles, i.e. $k_{i}=1$ and $K_{\sigma}=n$,

$$
\mathcal{Z}_{R_{Y_{n}}}=\frac{\chi_{R_{Y_{n}}}\left(\left(1^{n}\right)\right)}{n !} Z_{0}(f(\tilde{\beta}) N)^{n}+\mathcal{O}\left(N^{n-1}\right)=\frac{d_{Y_{n}}}{n !} Z_{0}(f(\tilde{\beta}) N)^{n}+\mathcal{O}\left(N^{n-1}\right)
$$

where $d_{Y_{n}}$ is the dimension of the representation of symmetry group given by the Young tableau $Y_{n}$ and the factorisation property of correlation functions in the large $\mathrm{N}$ limit is taken into account. Note, that we used the Frobenius relation for $S U(N)$ group and equation (38) for $U(N)$, since we expect that this difference can be neglected in the large $\mathrm{N}$ limit. $\operatorname{dim}_{R}$ being the character of the unit matrix also has the similar asymptotic behaviour for the large $\mathrm{N}$

$$
\operatorname{dim}_{R_{Y_{n}}}=\frac{d_{Y_{n}}}{n !} N^{n}+\mathcal{O}\left(N^{n-1}\right)
$$

The explicit formula for $1 / N$ corrections to (42) can be written in terms of the lengths $n_{i}$ of the Young tableau 13.

Therefore for $n$ fixed we get

$$
Z_{R_{Y_{n}}}=Z_{0}(f)^{n}+\mathcal{O}\left(N^{-1}\right) .
$$

Note that (43) for large $\tilde{\beta}$, fixed $n$ and large $\mathrm{N}$ is in agreement with well-known answer 114 $Z_{R}=1-C_{2}(R) / 2 N \tilde{\beta}$, where $C_{2}(R)$ is a quadratic Casimir.

Representing the sum over all representations in (28) as

$$
\mathcal{K}\left(U_{0}, U_{L}\right)=\sum_{n} \sum_{R \in Y_{n}} \operatorname{dim}_{R}\left(Z_{R}\right)^{(L-1)} \chi_{R}\left(U_{0} U_{L}^{+}\right),
$$

where the second sum in (44) is taken over all representations whose Young tableaux are in the set of Young tableaux with $n$ boxes, we have

$$
\mathcal{K}\left(U_{0}, U_{L}\right)=\left(Z_{0}\right)^{L^{\prime}} \sum_{n}(f)^{n L^{\prime}} \sum_{R \in Y_{n}} \operatorname{dim}_{R} \chi_{R}\left(U_{0} U_{L}^{+}\right)
$$


Using the Frobenius relation once again we represent $\mathcal{K}\left(U_{0}, U_{L}\right)$ for large $\mathrm{N}$ as

$$
\mathcal{K}\left(U_{0}, U_{L}\right)=\left(Z_{0}\right)^{L^{\prime}} \sum_{n} \frac{f^{n L^{\prime}}}{n !} \sum_{R \in Y_{n}} \operatorname{dim}_{R} \sum_{\sigma \in S_{n}} \chi_{R}(\sigma) \prod_{j=1}^{K_{\sigma}} \operatorname{tr}\left(U_{0} U_{L}^{+}\right)^{k_{j}}
$$

From this formula it is clear that the effective action describing an interaction of the field living on two infinite two-dimensional planes has all powers on $\tilde{U}_{0, z ; i} \tilde{U}_{L, z ; i}^{+}$. If we are interested in the first term of the effective action which is linear on $\tilde{U}_{0, z ; i}$ we can keep in (46) the permutation with cycles $\left(1^{n}\right)$ and we have

$$
\mathcal{K}\left(U_{0}, U_{L}\right)=\left(Z_{0}\right)^{L^{\prime}} \sum_{n} \frac{f^{n L^{\prime}}}{n !} \sum_{R \in Y_{n}} \operatorname{dim}_{R} \chi_{R}\left(\left(1^{n}\right)\right)\left(\operatorname{tr}\left[U_{0} U_{L}^{+}\right]\right)^{n}+\ldots
$$

Dots denote the terms with higher power of $U_{0} U_{L}^{+}$. Taking into account (42) at large $\mathrm{N}$ we have

$$
\mathcal{K}\left(U_{0}, U_{L}\right)=\left(Z_{0}\right)^{L^{\prime}} \sum_{n} \frac{f^{n L^{\prime}}}{n ! n !} \sum_{Y_{n}} d_{Y_{n}}^{2}\left(\operatorname{tr}\left[U_{0} U_{L}^{+}\right]\right)^{n}+\ldots
$$

Recall that the sum in (48) is taken over all Young tableaux with $n$ boxes. Due to the relation

$$
\sum_{Y_{n}} d_{Y_{n}}^{2}=n !
$$

we have for the transition function

$$
\mathcal{K}\left(U_{0}, U_{L}\right)=\left(Z_{0}\right)^{L^{\prime}} \exp \left\{f^{L^{\prime}} N \operatorname{tr}\left(U_{0} U_{L}^{+}\right)+\ldots\right\}
$$

Therefore for the large $\mathrm{N}$ and small $g^{2}$ the transition function can be represented as

$$
\mathcal{K}\left(U_{0}, U_{L}\right)=(2 \tilde{\beta})^{-\frac{L^{\prime}}{2 N^{2}}} \exp \left\{-\frac{3}{4 N^{2}} L^{\prime}+\left(1-\frac{1}{4 \tilde{\beta}}\right)^{L^{\prime}} N \operatorname{tr}\left(U_{0} U_{L}^{+}\right)+\ldots\right\} .
$$

In the strong coupling regime we have

$$
\mathcal{K}\left(U_{0}, U_{L}\right)=\exp \left\{-N^{2} L^{\prime} \tilde{\beta}(2-\tilde{\beta})+\tilde{\beta}^{L^{\prime}} N \operatorname{tr}\left(U_{0} U_{L}^{+}\right)+\ldots\right\} .
$$

Substituting the representations for the transition function in (27) we get

$$
\begin{aligned}
Z_{2+1}=\left(\tilde{Z}_{0}\right)^{L^{\prime} L^{2}} \int & \exp \left\{\left(1-\frac{L^{\prime}}{4 \tilde{\beta}}\right) N \sum_{i, z} \operatorname{tr}\left[V_{z} U_{0, z ; i} V_{z+i}^{+} \Omega_{z+i} U_{L, z ; i} \Omega_{z}^{+}\right]\right\} \\
& \times \prod_{z} d V_{z} d \Omega_{z}\left(\prod_{i} d U_{0, z ; i} d U_{L, z ; i}\right)
\end{aligned}
$$

for large $\tilde{\beta}$ and

$$
\begin{aligned}
Z_{2+1}=\left(\tilde{Z}_{0}\right)^{L^{\prime} L^{2}} & \int \exp \left\{(\tilde{\beta})^{L^{\prime}} N \sum_{i, z} \operatorname{tr}\left[V_{z} U_{0, z ; i} V_{z+i}^{+} \Omega_{z+i} U_{L, z ; i} \Omega_{z}^{+}\right]\right\} \\
& \times \prod_{z} d V_{z} d \Omega_{z}\left(\prod_{i} d U_{0, z ; i} d U_{L, z ; i}\right)
\end{aligned}
$$


for small $\tilde{\beta}$. The constant $\tilde{Z}_{0}$ is different in the different regimes.

It seems reasonable to assume that in the week coupling regime

$$
L^{\prime}=\log s
$$

Performing in the lattice effective action the limit $a \rightarrow 0$ one immediately recognize an action similar to the action (24) with special form of matrix $M$,

$$
M^{A B}=\left(\begin{array}{ll}
0 & \delta \\
\delta & 0
\end{array}\right), \quad \delta=1-\frac{1}{4 \tilde{\beta}} \log s,
$$

i.e.

$$
\left.S\left[V, \Omega, A_{i}\right]=\left(1-\frac{L^{\prime}}{4 \tilde{\beta}}\right) N \int d^{2} z \operatorname{tr}\left[V(z) D_{i}^{-} V^{+}(z) \Omega_{(} z\right) D_{i}^{+} \Omega^{+}(z)\right]
$$

where

$$
D_{i}^{ \pm}=\partial_{i}+A_{i}^{ \pm}, \quad U_{L, z ; i}=e^{a A_{i}^{+}(z)}, \quad U_{0, z ; i}=e^{a A_{i}^{-}(z)}
$$

In this example the analog of quark-quark scattering amplitude is given by the expectation value of two longitudinal Wilson lines

$$
\begin{aligned}
& \mathcal{A}=\int d^{2} z e^{i q z}<\mathcal{V}(0) \mathcal{V}^{+}(z)> \\
& \mathcal{V}(z)=\operatorname{tr}\left[P \exp \int d y A(y, z)\right]
\end{aligned}
$$

The lattice version of $(60)$ is simply

$$
\mathcal{V}_{z}=V_{0, z} V_{L, z}^{+}
$$

Note that in the model example of the one-dimensional transversal plane one can perform the integration over fields $d V_{z} d \Omega_{z}$ explicitly. Indeed, taking into account the form (28) for the transition function $\mathcal{K}\left(U_{0}, U_{L}\right)$

$$
\begin{gathered}
Z_{1+1}=\int \prod_{z=1}^{M} \mathcal{K}\left(V_{z} U_{0, z} V_{z+i}^{+}, \Omega_{z} U_{L, z} \Omega_{z+i}^{+}\right) d V_{z} d \Omega_{z} d U_{0, z ; i} d U_{L, z ; i} \\
=\int \prod_{z=1}^{M} \sum_{R} \operatorname{dim}_{R}\left(Z_{R}\right)^{(L-1)} \chi_{R}\left(V_{z} U_{0, z} V_{z+i}^{+} \Omega_{z+i} U_{L, z}^{+} \Omega_{z}^{+}\right) d V_{z} d \Omega_{z} d U_{0, z ; i} d U_{L, z ; i}, \quad i=1,
\end{gathered}
$$

and the orthogonality condition for characters we have

$$
Z_{1+1}=\int \sum_{R}\left(Z_{R}\right)^{(L-1)(M-1)} \chi_{R}\left(\Omega^{+} V_{1} P_{+} \prod_{z} U_{0, z ; i} V_{M}^{+} \Omega_{M} P_{-} \prod_{z} U_{L, z ; i}^{+}\right) d U_{0, z ; i} d U_{L, z ; i}
$$

Here $P_{+}$and $P_{+}$are ordering and antiordering products. This answer was obvious from the beginning since in this case we deal with the two-dimensional QCD [15, 16]. 


\section{Concluding Remarks and Discussion}

Let us make some comments about the 4-dimensional case. In this case the y-lattice is two-dimensional and we have to deal with the following lattice integrals

$$
\begin{gathered}
Z_{2+2}=\int \prod_{(z, i)} \int \exp \left\{\frac{a N}{4 g^{2}} \sum_{y, \alpha} \operatorname{tr}\left(\tilde{U}_{y, z ; i} \tilde{U}_{y+\alpha, z ; i}^{+}+\tilde{U}_{y+\alpha, z ; i} \tilde{U}_{y, z ; i}^{+}-2\right)\right\} \\
\times \quad \prod_{\tilde{U}_{y_{1}, L_{2}, z ; i}=V_{y_{1}, L_{2}, z} U_{y_{1}, L_{2}, z ; i} V_{y_{1}, L_{2}, z+i}^{+}} d \tilde{U}_{y, z ; i} \\
\tilde{U}_{y_{1}, 0, z ; i}=V_{y_{1}, 0, z} U_{y_{1}, 0, z ; i} V_{y_{1}, 0, z+i}^{+}, \tilde{U}_{L_{1}, y_{2}, z ; i}=V_{L_{1}, y_{2}, z} U_{L_{1}, y_{2}, z ; i} V_{L_{1}, y_{2}, z+i}^{+}, \\
\tilde{U}_{0, y_{2}, z ; i}=V_{0, y_{2}, z} U_{0, y_{2}, z ; i} V_{0, y_{2}, z+i}^{+}, \tilde{y}_{y_{1}, 0, z ; i} d U_{y_{1}, L_{2}, z ; i} d U_{L_{1}, y_{2}, z ; i} d U_{0, y_{2}, z ; i} d V_{y_{1}, 0, z} d V_{y_{1}, L_{1}, z} d V_{L_{1}, y_{2}, z} d V_{0, y_{2}, z} \\
\times d U_{y_{1}}
\end{gathered}
$$

In (64) we have the product of the transition functions of the two-dimensional lattice chiral model. If we assume the periodic boundary conditions in one of two longitudinal direction, say $y_{2}$-direction,one can expect that for $L_{1} \gg L_{2}$ the main contribution comes from the one dimensional excitations, i.e.

$$
\begin{gathered}
Z_{2+2} \sim \prod_{y_{2}}\left(Z_{1+2}\right)=\left(\tilde{Z}_{0}\right)^{\operatorname{vol}_{4}} \\
\times \int \exp \left\{\left(1-\frac{\log s}{4 \tilde{\beta}}\right) N \sum_{i, z, y_{2}} \operatorname{tr}\left[V_{y_{2}, z} U_{0, y_{2}, z ; i} V_{y_{2}, z+i}^{+} \Omega_{y_{2}, z+i} U_{L, y_{2}, z ; i} \Omega_{y_{2}, z}^{+}\right]\right\} \\
\times \prod_{z}\left(d V_{y_{2}, z} d \Omega_{y_{2}, z}\left(\prod_{i} d U_{0, y_{2}, z ; i} d U_{L, y_{2}, z ; i}\right)\right)
\end{gathered}
$$

In the continuous version the contribution of long one-dimensional tubes apparently is still described by the action (57), in spite of the summation over $y_{2}$ in (65), i.e.

$$
\begin{gathered}
Z_{2+2} \sim\left(\tilde{Z}_{0}\right)^{v o l_{4}} \int \exp \left\{\left(1-\frac{\log s}{4 \tilde{\beta}}\right) N \int d^{2} z \operatorname{tr}\left[V(z) D_{i}^{-} V^{+}(z) \Omega(z) D_{i}^{+} \Omega^{+}(z)\right\}\right. \\
\times \prod_{z} d V(z) d \Omega(z)\left(\prod_{i=1,2} d A_{i}^{+}(z) d A_{i}^{-}(z)\right),
\end{gathered}
$$

Equation (65) describes a rough approximation, however it gives a rather acceptable physical picture. This picture is in agreement with the Verlinde and Verlinde answer which is in accordance with perturbation calculations [2] and has the shock-wave [17, 18, 19] semiclassical interpretation [4].

Let us stress that the question about dominate contributions in (65) or in others words the question about the behaviour of non-linear $\sigma$ model with non-trivial boundary conditions is rather complicated and without doubt it is worthy more attention.

The Lagrangians (14) and (7) show a relation between the 4-dimensional YangMills theory and the two-dimensional chiral model. A relation was expected long time ego and was motivated by the fact that the both theories have dimensionless coupling constant and both are asymptotically free. Note also that some similarity was expected between the usual local formulation of the two-dimensional chiral model and the loop formulation, i.e. dynamics of long tubes excitations in high-dimensional 
Yang-Mills theory [20, 21]. The Verlinde and Verlinde truncated action [4] together with the assumption (65) make this expected relation more tangible.

In concluding, it has been argued that consideration of quantum fluctuations of one-

dimensional excitations confirms the two-dimensional picture of high-energy scattering in QCD.

\section{ACKNOWLEDGMENT}

The author is grateful to G.Arutyunov and K.Zarembo for useful discussions.

\section{References}

[1] L.N. Lipatov, Review in Perturbative QCD, ed. A.H. Mueller (World Scientific, Singapore, 1989), and references therein.

[2] H. Cheng and T.T. Wu, Expanding Protons: Scattering at High Energies, (The MIT Press, Cambridge, Massachusetts), and references therein.

[3] L.N. Lipatov, Nucl.Phys. B365 (1991) 614.

[4] H.Verlinde and E.Verlinde, QCD at High Energies and two-dimensional field theory, preprint, PUPT-1319, IASSNS-HEP-92/30, hep-th/9302104, February,1993.

[5] Mc.Kane and M.Stone, Nucl.Phys. B163 (1980) 169;

E.Brezin,Nucl.Phys. B166 (1980) 588.

[6] A.T.Ogielski, Phys.Rev. 25 (1982) 2634.

I.Bars, M.Gunaydin and S.Yankelowich, Nucl.Phys. 219(1983)81.

[7] M.Caselle, A.D'Adda and S.Panzeri, Phys. Lett. 302B(1992)80.

[8] H.Weyl, The Classical Groups, Princeton U.P.,1946.

[9] I.Bars, J.Math. Phys 21 (1980) 2678.

[10] S.Samuel, J.Math. Phys 21 (1980)

[11] D.Gross and E.Witten, Phys. Rev. D21 (1980)446 .

[12] E.Brezin and D.Gross, Phys. Lett. 97B(1980)120.

[13] D.Gross and Taylor, Two Dimensional QCD is a String Theory, preprint PUPT-1376, LBL-33458, UCB-PTH-93/02, January, 1993.

[14] J.-M.Drouffe and J.-B.Zuber, Phys. Rep. 102 (1983) 1.

[15] A.Migdal, JETP 69 (1975) 810.

[16] B.Rusakov, Mod.Phys.Lett. 5 (1990)693 
[17] G. 't Hooft, Phys. Lett. B198 (1987) 61; Nucl. Phys. B304(1988) 867.

[18] I. Munizich and M. Soldate, Phys. Rev. D 37, 353 (1988).

D. Amati, M. Ciafaloni and G. Veneziano, Int. J. of Mod. Phys. 3 (1988) 1615; CERN preprint (Februari 1992)

[19] G. de Vega and N.Sanches Phys.Lett. B (1990)

[20] A.Polyakov, Phys.Lett. B82 (1979) 247.

[21] I.Aref'eva , Lett.Math.Phys. 3 (1979) 241. 